\title{
Descentralización de la oferta turística como estrategia de competitividad urbana. Programas "de barrio" en Ciudad de México y Ciudad Autónoma de Buenos Aires
}

\author{
Luciana Mabel Rodriguez* \\ CONICET-Instituto de Geografía (Argentina)
}

\begin{abstract}
Resumen: El artículo propone indagar, de manera comparativa, sobre una serie de instrumentos de la política pública orientados al desarrollo de la actividad turística en las Ciudades de México y Buenos Aires en años recientes. El interés del trabajo radica en mostrar que, aunque se trata de ciudades con orientaciones políticas diferentes, sus estrategias discursivas y materiales poseen características similares: el turismo como medio de competitividad en el mercado internacional y recurso para el desarrollo económico, social y cultural, a través de su descentralización en barrios alejados de los circuitos tradicionales, en miras de incrementar la oferta turística. Entendiendo al turismo como una actividad que, de manera creciente, cristaliza criterios de gobernanza empresarial, el articulo demuestra como este modelo se impone sobre las particularidades locales, legitimando posibles acciones de valorización urbana en donde los gobiernos locales adquieren el rol de promotores de la oferta privada.
\end{abstract}

Palabras Clave: Turismo urbano; Política pública; Competitividad urbana; Ciudad de México; Ciudad autónoma de Buenos Aires.

Tourist offer decentralization as urban competitiveness strategy. "Neighborhood" programs in Mexico City and the Autonomous City of Buenos Aires

Abstract: The article is a comparative study of a series of recent public policy instruments aimed at the development of tourism in the City of Mexico and Buenos Aires. The interest of the work lies in its revealing that although these two cities have different political orientations, the strategies and structures used are essentially similar, aimed at making tourism into a competitive resource for economic, social and cultural development, through its de-centralisation in neighborhoods far from the traditional circuits, in order to augment the possibilities for tourism on offer. It works from the growing business perspective of tourism as a local participatory process, of governance, and shoes how this model can be applied in very different places, allowing for possible intervention of local authorities in the re-valorisation of their area as private promoters of tourism.

Keywords: Urban tourism; Public policy; Urban competitiveness; Mexico city; Autonomous city of Buenos Aires.

\section{Introducción}

Desde el momento en que el turismo se incorporó en las agendas públicas de México y Argentina, ha sido clave su rol como recurso para la construcción de cierta imagen de lugar. Un medio a través del cual se difundían atributos sobre el país que se quería mostrar al exterior, pero también hacia la propia ciudadanía. Así, acompañando la idea de progreso en ambos países, el turismo estuvo inicialmente ligado a la idea de elemento civilizador en oposición al período de la Revolución Mexicana o instando a la población a recorrer y familiarizarse con el extenso territorio, en el caso de Argentina. Su epicentro: las ciudades capitales, que hacia la segunda mitad del siglo XX se constituían como las puertas de ingreso a sus países, encarnando la imagen de modernidad y progreso que se quería difundir para el mundo.

CONICET-Instituto de Geografía (Argentina); E-mail: arqlucianarodriguez@gmail.com 
Actualmente, sin embargo, la concepción positiva de la actividad turística ya no se presenta como sinónimo de civilidad y progreso en términos discursivos, sino más bien, como un recurso que permite palear problemas urbanos como desempleo, deterioro ambiental o inseguridad. Un medio que permite mejorar la calidad de vida de los habitantes a partir del supuesto derrame económico que la actividad genera; al tiempo que persiste su empleo para la construcción de una imagen de lugar, aunque ahora asociada a atributos de competitividad global: ciudad cosmopolita, creativa, sustentable, diversa, etc. Entre otras, una estrategia se presenta en las agendas gubernamentales de ambos países como el medio para conseguirlo: la diversificación de la oferta turística a través de su descentralización, propiciando el contacto con las tradiciones o espacios locales auténticos. Idea que suele asociarse también al fortalecimiento de la identidad local.

Como punto de partida para este trabajo, a nivel de las ciudades capitales de México y Argentina, advertimos que esta meta encuentra su correlato espacial en la promoción de áreas urbanas alejadas de los centros tradicionales, y en la capitalización de ciertas prácticas o actividades allí desarrolladas (desde festividades religiosas hasta actividades comerciales), a través del desarrollo de programas análogos. Una dinámica que podemos enmarcar en los cambios que las políticas urbanas de las Ciudades de México y Buenos Aires vienen experimentando durante las últimas décadas, de la mano de formas de gobernanza orientadas hacia criterios empresariales. Así, cristalizando los principales lineamientos de la planificación estratégica (el modelo de gestión empresarial más difundido en las administraciones públicas), encontramos estrategias convergentes en ambas ciudades basadas en criterios de competitividad, gran protagonismo del sector privado y flexibilización de los instrumentos de gestión. De esta forma, tal como señala Delgadillo (2014), aunque sus gobiernos corresponden a orientaciones políticas distintas, impulsan planes y programas urbanos con evidentes similitudes.

En este sentido, el artículo propone contribuir al entendimiento de las estrategias adoptadas en relación a la descentralización de la oferta turística -específicamente aquellas orientadas a promover sitios de carácter barrial como forma de conectar a los visitantes con espacios y prácticas locales-, en las Ciudades de México y Buenos Aires. Dos ciudades constituidas como las principales receptoras de turistas respecto de sus sistemas nacionales y que, además de presentar caminos comparables de autonomía política, ilustran también muchos de los retos sociales, económicos y físicos de las grandes ciudades contemporáneas (Kanai y Ortega-Alcázar, 2009).

A tal fin, en primer lugar, se establece el marco teórico que estructura el desarrollo del trabajo y la metodología que lo sustenta. En segundo lugar, se analizan los planes y programas de turismo desarrollados en las últimas tres décadas, tanto a nivel nacional como local, con el objetivo de comparar sus discursos y comprobar su vinculación con criterios de gobernanza empresarial. Luego se estudian, de manera específica, los proyectos vinculados a la temática de turismo en barrios, entendidos como estrategias de descentralización de la oferta que permiten contrastar discursos y estrategias espaciales. Finalmente se exponen las conclusiones del trabajo.

\section{Competitividad y turismo urbano}

Con el paso del gerencialismo al empresarialismo urbano (Harvey, 1989), el turismo se volvió un recurso para la competitividad entre ciudades, en correspondencia con los objetivos del modelo propuesto por la planificación estratégica. Con esto nos referimos a una forma de gobernanza que prioriza las alianzas público-privadas de carácter empresarial, porque su ejecución y diseño son especulativos -están sujetos a los vaivenes del mercado-, donde los gobiernos locales ganan el rol de "socios estratégicos" de negocios (Jessop, 2002:454), en contraste con el planeamiento racional de las décadas anteriores. Desde este paradigma, la gestión de las ciudades adopta criterios empresariales de competitividad, atractividad, equidad territorial, eficiencia y participación ciudadana, como principios rectores de las políticas urbanas (Borja y Castells, 1997; Fernández Güell, 1997).

Amparada en la visión positiva del turismo como impulsor del desarrollo -legitimándose en discursos que apelan a su capacidad de generar empleo, atraer divisas internacionales o disminuir las desigualdades regionales (Almirón et al., 2008)-, ${ }^{1}$ fundamento discursivo base de las estrategias de gestión, la actividad se convirtió en un nuevo recurso económico para enfrentar la crisis en las ciudades posindustriales (Harvey, 1998; Smith, 1996; Baum, 1994; Britton, 1991, entre otros). Así, bajo el modelo de gobernanza mencionado, marketing urbano (incluso utilizado como herramienta de marketing político), ${ }^{2}$ declaratorias patrimoniales, rankings internacionales, nominaciones a eventos, redes de ciudades y planes orientativos 
que cristalizan buenas prácticas globales, entre otros, se volvieron los recursos destacados para el logro de la competitividad urbana (y turística) entre las ciudades.

Una dinámica que termina por inscribirse en la reducción de la intervención directa del Estado en la oferta turística, con un mayor énfasis en la colaboración público-privada (Hall, 2000). Esto se traduce, a su vez, en la limitación en el empleo de los instrumentos normativos de la política pública y, tendencialmente, en la desregularización de la actividad turística en general. Como menciona Vainer (2000), enmarcándose en el concepto de ciudad-mercancía, las estrategias no vinculantes se presentan como las más adecuadas, en la medida que reducen el control estatal y permiten que la ciudad se adapte flexiblemente a las tendencias del mercado. De esta forma, se soslayan políticas urbanas clásicas asociadas a planes normativos y visiones urbanas globales, que desde la perspectiva de la planificación estratégica se consideran obsoletas porque no permiten respuestas rápidas a las nuevas demandas del mercado (Vainer, 2000).

Acompañando esta tendencia de las políticas públicas, presenciamos que a las estrategias de turismo que han caracterizado las intervenciones en las ciudades durante las últimas décadas (activación patrimonial de edificios, creación de hitos urbanos o embellecimiento del espacio público en centros históricos) se incorpora la promoción de sitios novedosos, alejados de los circuitos tradicionales. Bajo discursos que apelan a su capacidad de generar experiencias auténticas ${ }^{3}$ para aquellos turistas interesados en involucrarse con la vida de los locales, ciertas áreas urbanas son valorizadas como nuevos atractivos turísticos. Un fenómeno que viene siendo analizado desde el lado de la demanda -especialmente a partir de la valorización de turistas, trabajadores y residentes- (Maitland, 2008; Maitland y Newman, 2009; Novy, 2011), aunque de forma más acotada desde el lado de la oferta, concretamente a partir de las políticas públicas de turismo. Es decir, a partir de lo que el Gobierno decide hacer o no hacer en relación específica a la actividad (Hall, 2010).

En este marco, durante la transición a los años 90, las ciudades de México y Buenos Aires comenzaron a experimentar transformaciones en relación con su forma de administración. Específicamente, el reacondicionamiento de sus herramientas para consolidar un modelo de gestión estatal orientado al empresarialismo urbano. Algo que, entre otras cosas, se vio reflejado en el desarrollo de planes y programas que asumen la competitividad de estas ciudades como uno de sus objetivos principales, en el marco de la descentralización de funciones del Estado y el cambio en el estatus jurídico de las mismas. ${ }^{4}$ Caminos análogos, aunque bajo ideologías políticas diferentes que se fueron consolidando en los últimos años: el Partido de la Revolución Democrática (PRD) de centro-izquierda y Partido Morena de izquierda en Ciudad de México; y la Propuesta Republicana (PRO) de derecha en Ciudad de Buenos Aires.

Retomando a Delgadillo (2014), incluso en el contexto de orientaciones políticas diferentes, en ambas ciudades se vienen impulsando políticas públicas y programas urbanos similares, orientados a construir cierta imagen urbana e incrementar las ventajas competitivas en el mercado internacional. Siguiendo esta línea, en el caso del turismo se desarrollan estrategias de descentralización de la oferta que aprovechan los rasgos de autenticidad asociados a determinados barrios como recursos para incrementar la oferta turística (aunque no se desconocen otras iniciativas que promueven la descentralización del turismo en estas ciudades). En este sentido, nos preguntamos ¿cuáles son los objetivos de los planes y programas de turismo desarrollados a nivel nacional y local en las últimas tres décadas? ¿Qué tienen en común sus discursos con criterios de gobernanza empresarial y cómo se vinculan con estrategias de descentralización? ¿Cómo son los proyectos desarrollados bajo la temática de turismo en barrios? ¿Existe un correlato entre los discursos enunciados en los planes y programas y sus estrategias materiales?

Respecto a los antecedentes sobre el tema, aunque contamos con algunos trabajos que abordan las políticas públicas de turismo en países latinoamericanos de manera comparada (Balslev Clausen y Velázquez, 2013; Barbibni et al., 2011; Bowman, 2013, 2014; Schenkel, 2017; Wallingre, 2017), no se encuentran registros de un abordaje que las ponga en relación a escala de las Ciudades de México y Buenos Aires. En este sentido, cabe señalar que, desde el enfoque de las políticas públicas, el turismo permanece como una pequeña sub-área de estudio, a pesar de que existen numerosos ejemplos de cómo los cambios en la política afectan sus patrones, procesos y direcciones (Hall, 2010). Tal como indica Velasco (2011), existen pocas investigaciones sobre el desarrollo de políticas turísticas, por lo que se carece de pensamiento crítico suficiente que permita superar la etapa de investigación inicial en la materia; algo que, de acuerdo con la autora, tiene estrecha relación con la primacía de la perspectiva económica en los estudios de turismo.

Atendiendo a esta vacancia temática, y fundamentado en la importancia que el turismo ha adquirido en las agendas públicas urbanas, el trabajo se orientará a responder los interrogantes expuestos a partir del análisis de ciertos instrumentos de la política pública. Sin desconocer que la elaboración de dichas 
políticas está sujeta a redes de actores públicos y privados que interactúan en distintos ámbitos y escalas (Jacint, 2011), el estudio se acotará al análisis de los discursos y estrategias territoriales enunciadas en los diferentes planes y programas, reparando en su vinculación con criterios de gobernanza empresarial.

\section{Metodología}

Para el desarrollo del trabajo se empleó una metodología exploratoria-descriptiva, de enfoque cualitativo, basada en dos momentos. En el primero, se realizó una sistematización y análisis comparado de instrumentos de la política pública orientados al fomento del turismo, en las Ciudades de México y Buenos Aires y sus respectivos contextos nacionales. Específicamente, se abordaron aquellos planes y programas generados durante las últimas tres décadas (coincidiendo con los cambios en la forma de gestión de ambas ciudades), junto con su marco legislativo. Para su estudio, los documentos fueron codificados bajo las categorías de análisis afines a competitividad, diversificación, descentralización de la oferta turística y desarrollo económico.

En el segundo momento, el estudio se enfocó en estrategias recientes orientadas al desarrollo de la actividad turística en barrios de ambas ciudades. A través de su análisis comparativo, se discriminaron los principales lineamientos que los sustentaban y su respectivo correlato espacial a través de diferentes mapeos (Figuras 1-4). Trabajo que se completó con el relevamiento de noticias periodísticas y la realización de entrevistas semi-estructuradas a informantes clave pertenecientes a los organismos de turismo locales: la Dirección de Desarrollo de Proyectos Turísticos y la Dirección General del Instituto de Promoción Turística de la Secretaria de Turismo de la Ciudad de México (en junio de 2019), y la Gerencia Operativa de Herramientas Digitales y Desarrollo de la Oferta del Ente de Turismo de la Ciudad de Buenos Aires (en octubre de 2019).

\section{Los instrumentos de gestión de los gobiernos locales}

De acuerdo con Sánchez y Gutiérrez (2016), a partir de la década del 90 comienza la descentralización de las funciones de la Secretaría de Turismo de México (SECTUR), estimulándose la participación de los gobiernos estatales y del sector privado, con menor intervención del Estado. Basada en una concepción del turismo como recurso para elevar el nivel de vida económico, social y cultural de los habitantes, en el año 1992 se sanciona la nueva Ley Federal de Turismo. Esta Ley estableció la creación de un órgano encargado de las funciones turísticas en cada entidad federativa y en los municipios turísticos, y dio paso también a la transferencia de diferentes funciones del sector.

De la mano de los sucesivos Planes Nacionales de Desarrollo ${ }^{5}$ y sus respectivos Programas Sectoriales de Turismo (1995-2000, 2001-2006, 2007-2012, 2013-2018), se fueron consolidando los discursos de diversificación y descentralización de la oferta. En este sentido, dentro del diagnóstico del último Programa Sectorial de Turismo 2013-2018, se señalaba como "factores que debilitan la competitividad del sector turístico" a la concentración de la oferta del país (deficiente distribución territorial), lo que también impactaba en la poca captación de segmentos de mercado (SECTUR, 2013_a, s/n). Al mismo tiempo, entre los objetivos del programa se instaba a "fortalecer las ventajas competitivas de la oferta turística" a través de estrategias de diversificación, en cuyos lineamientos la "identidad" (aunque sin mayores especificaciones) se presentaba como parte del modelo de desarrollo turístico deseable (SECTUR, 2013_a, s/n).

En la esfera local, varios años después de las primeras elecciones del Distrito Federal en 1997, se sanciona la Ley de Turismo del Distrito Federal de 2010, que estableció las funciones de su Secretaría de Turismo (SECTUR DF o SECTUR CDMX). ${ }^{6}$ Entre los principales lineamientos de la Ley, también se mencionaba el "fortalecimiento de la competitividad turística del Distrito Federal". Aunque sin alusión explícita a la descentralización de la oferta, sí incorporaba como instrumento a las "Zonas de Desarrollo Turístico Local Prioritarias", entendidas como aquellas áreas que por sus "características naturales, ecológicas, históricas o culturales, constituyan un atractivo turístico que coadyuve al crecimiento económico de una zona, o bien, aquella que cuente con la potencialidad para desarrollar actividades turísticas" (Ley de Turismo del Distrito Federal, 2010). En el Reglamento de esta Ley (2012), las Zonas de Desarrollo encuentran un correlato directo, al menos en su definición, con los "Barrios Mágicos" (programa que se explicará más adelante). 
Esta Ley y su Reglamento, fueron los sustentos legislativos principales del último Programa Sectorial de Turismo 2013-2018 de la CDMX, elaborado bajo criterios de gobernanza empresarial (competitividad, participación ciudadana y cooperación público-privada), con alusión directa a su flexibilidad "para enfrentar las circunstancias cambiantes de una gran comunidad" como la Ciudad de México (SECTUR, 2013_b, p. 11). Basándose en la concepción del turismo como motor de progreso y fuente de divisas, el documento retoma los principales resultados de la Agenda de Competitividad Turística de 2013, estableciendo la necesidad de diversificar la oferta turística en todas las zonas que integran el Distrito Federal, en pos de aumentar la competitividad a través de productos y experiencias únicas y singulares (SECTUR, 2013_b). En este sentido, dentro de sus ejes estratégicos, plantea la ampliación de la oferta turística a través del desarrollo y fomento de nuevos productos, y la redistribución territorial de la actividad en pos de desarrollar "una ciudad compacta, dinámica, policéntrica y equitativa" (SECTUR, 2013_b, p.58).

Por su parte, en Argentina, la nueva Ley Nacional de Turismo se sancionada en 2004. En sus dos primeros artículos contempla aspectos similares a la Ley Federal de Turismo mexicana, entre otros: la contribución al desarrollo social, económico y cultural de la actividad, aunque con mayor énfasis en su carácter estratégico y en el rol destacado del sector privado (Ley Nacional de Turismo, N²5997, 2005). En el marco de esta Ley, se crea el Instituto de Promoción Turística (INPROTUR), un ente público-privado encargado de desarrollar programas y estrategias de fomento del turismo receptivo internacional, a través de la promoción de la marca pais, con el objetivo de “(...) difundir nacional e internacionalmente los factores diferenciales de nuestro país a partir de cuatro escenarios comunicacionales: turismo, cultura, inversiones y comercio internacional" (INPROTUR, 2016, s/n).

Apoyado en este marco normativo, en 2005 se elaboró la primera versión del Plan Federal Estratégico de Turismo Sustentable a nivel nacional con proyección a $2016,{ }^{7}$ impulsado por la Secretaría de Turismo de la Nación, bajo el objetivo principal de fijar las estrategias de desarrollo turístico de Argentina. Basado también en la participación ciudadana y el asociacionismo público-privado ("fortalecimiento del tejido empresarial nacional"), dentro de los bloques que organizaron el plan, cabe destacar el "desarrollo equilibrado del espacio turístico nacional" a través de la consolidación de destinos y productos actuales y la promoción de aquellos emergentes (Secretaría de Turismo, 2005). En este sentido las estrategias definidas para el desarrollo de la oferta aludían, explícitamente, a la diversificación y descentralización de atractores, destinos y productos (Secretaría de Turismo, 2005).

En la Ciudad de Buenos Aires, fue recién con su autonomía en el año 1996 que se inicia una valoración diferente y positiva del turismo como actividad económica, cobrando aun mayor importancia después de la devaluación del año 2001 (Gómez y Zunino Singh, 2008). En ese año se creó la Ley de Turismo de la Ciudad Autónoma de Buenos Aires, ratificando la idea del posicionamiento de la ciudad como "producto turístico competitivo". Una Ley que, además, buscaba "satisfacer las expectativas del mercado a través de la búsqueda de nuevos recursos turísticos que contribuyan al enriquecimiento del patrimonio y a la diversificación de la oferta turística" (Ley de Turismo de la Ciudad Autónoma de Buenos Aires, $\mathrm{N}^{\circ}$ 600/01, 2001). En este sentido, y de manera análoga a las Zonas de Desarrollo Turístico mexicanas, propone la figura de "lugares turísticos de desarrollo prioritario" entendidos como aquellos sitios que, por sus características naturales, histórico-patrimoniales o culturales pueden constituir un atractivo (Ley de Turismo de la Ciudad Autónoma de Buenos Aires, №600/01, 2001).

Durante el primer año de gobierno de la Propuesta Republicana (PRO), en 2007 se crea el Ente de Turismo de la Ciudad (ENTUR), actualmente dependiente de la Secretaría General y Relaciones Internacionales. ${ }^{8}$ Con él comienza una importante estrategia de promoción orientada a "consolidar el crecimiento turístico, mejorar la estructuración de los productos turísticos, aumentar el ingreso en una proporción mayor al volumen de llegadas por turismo y diversificar las áreas turísticas de la ciudad" (Rodriguez et al., 2011: 109).

Así, la Ciudad de Buenos Aires se convirtió en objeto de una fuerte campaña de promoción turística encarada el gobierno local, orientada a reforzar el crecimiento del turismo receptivo y a atraer inversiones promoviendo una cierta imagen de ciudad. Desde este momento, comienzan a desarrollarse una serie de planes orientativos, aunque pocas veces específicos de turismo, que lo incluyen a partir de un espectro de temas más basto y de discursos sobre el posicionamiento, imagen y atributos competitivos de la ciudad: Plan Estratégico Buenos Aires 2010; Plan Estratégico Buenos Aires 2016, con perspectiva metropolitana y Plan Estratégico Participativo Buenos Aires $2035 .^{9}$

De acuerdo con lo expuesto, si bien en ambos casos existen diversos instrumentos orientados al desarrollo de la actividad turística, lo cierto es que, en lo referido a competitividad, diversificación y descentralización de la oferta y desarrollo económico, éstos generan pocas precisiones. Así, aunque 
notamos coherencia discursiva entre planes a nivel nacional y local, su generalidad admite un amplio rango de acciones para la concreción de los objetivos enunciados.

\section{El turismo llega a los barrios}

A continuación, nos proponemos analizar algunos programas análogos a ambas ciudades, en donde la idea de descentralización de la oferta turística aparece como respuesta a problemas socio-espaciales de la mano del fortalecimiento o rescate de la identidad local, a partir de la misma unidad territorial: el barrio.

\section{1. "Barrios Mágicos" y "Turismo de Barrio" en Ciudad de México}

En el año 2011, el SECTUR DF lanzó el Programa de Barrios Mágicos, que tomó como antecedente el difundido Programa Federal de Pueblos Mágicos iniciado en 2001. ${ }^{10}$ De acuerdo al Reglamento de la Ley de Turismo del Distrito Federal (2012), se entendía por Barrios Mágicos Turísticos a aquellas

(...) áreas territoriales de las Delegaciones que, por sus características naturales, ecológicas, históricas o culturales, poseen identidad propia, y cuyos habitantes tienen un sentido de pertenencia y comparten elementos culturales como mitos, leyendas, historias, hechos trascendentes y costumbres, que emanan de sus raíces y se manifiestan en sus relaciones sociales, identificándose como atractivos turísticos.

Así, con 21 barrios declarados, localizados principalmente en el centro-oeste de la ciudad (Figura 1), el programa pretendía descentralizar la oferta turística hacia sectores más alejados, apelando tanto a sus atributos materiales como inmateriales:

El objetivo general es ampliar la oferta turística de la Ciudad de México, mediante la creación de nuevos productos turísticos y mejorar la calidad de vida de los habitantes de cada Barrio Mágico Turístico, a través de la creación de empleos e incrementar la derrama económica, recuperar los espacios urbanos y desarrollar el tejido empresarial relacionado con el turismo. (SECTUR DF, 2011, p. 51).

A principios de 2019, con el inicio de la nueva gestión de la ciudad y siguiendo algunos de los lineamientos de aquel programa, ${ }^{11}$ se lanzó Turismo de Barrio. Se trata de un proyecto planteado a mediano y largo plazo, desarrollado por el SECTUR CDMX, con el objeto de generar nuevos productos turísticos en nueve alcaldías del sector oriente de la ciudad: Gustavo A. Madero, Venustiano Carranza, Iztacalco, Iztapalapa, parte de Coyoacán, Milpa Alta, Xochimilco y Tláhuac. De acuerdo con el Secretario de Turismo, Carlos Mackinlay Grohmann, es común a estas alcaldías una menor integración social, problemas de movilidad, inseguridad, deficiencia de espacios verdes y mayor desempleo que el resto de la ciudad; aunque cuentan con posibilidades para desarrollar "experiencias turísticas" relacionadas al encuentro del visitante con las formas de vida de la gente, el barrio, la gastronomía, los oficios, etc. (Rammsy, 08 de abril de 2019).

Lo que pretendemos es que los beneficios del turismo en la CDMX se vayan a las zonas que han sido marginadas del desarrollo turístico (...) donde no hay tanto patrimonio tangible ni oferta hotelera, pero sí una gran posibilidad de desarrollar ofertas turísticas ligadas a la vida del barrio, los oficios, talleres y la gastronomía. Eso es algo que busca el turismo nacional e internacional, ver cómo vive la gente y no sólo quedarse en un hotel. (Rammsy, 2019, 08 de abril).

De acuerdo con el titular de la Dirección de Desarrollo de Proyectos Turísticos (DDPT) del SECTUR CDMX, sobre el origen del programa:

Para contrarrestar un poco esto [inseguridad] hay que ser más incluyentes. Entonces, en ese "ser incluyente", una de las grandes políticas era: ¿cuáles son las zonas menos turísticas de la ciudad? El sur-sur y oriente (...) un sector urbano muy denso, muy interesante, pero también con condiciones que no son las mejores. (Arq. Victor Salas Castilla, comunicación personal, 20 de junio de 2019).

Para su ejecución, el SECTUR CDMX plantea asistencia técnica, capacitación y acompañamiento, a fin de "crear figuras barriales" a partir del desarrollo de nuevas habilidades y destrezas en la población local: cocineras tradicionales, promotores turísticos barriales de la gastronomía, ecoturismo, cultura, arte urbano, religión, etc. (SECTUR, 15 de marzo de 2019). Además del beneficio planteado para la población de las alcaldías -se menciona con frecuencia la reducción de la inseguridad-, también se fija la meta de aumentar el número de visitantes extranjeros en la ciudad (actualmente la composición es 
Figura 1: Localización de los programas Barrios Mágicos y Turismo de Barrio.

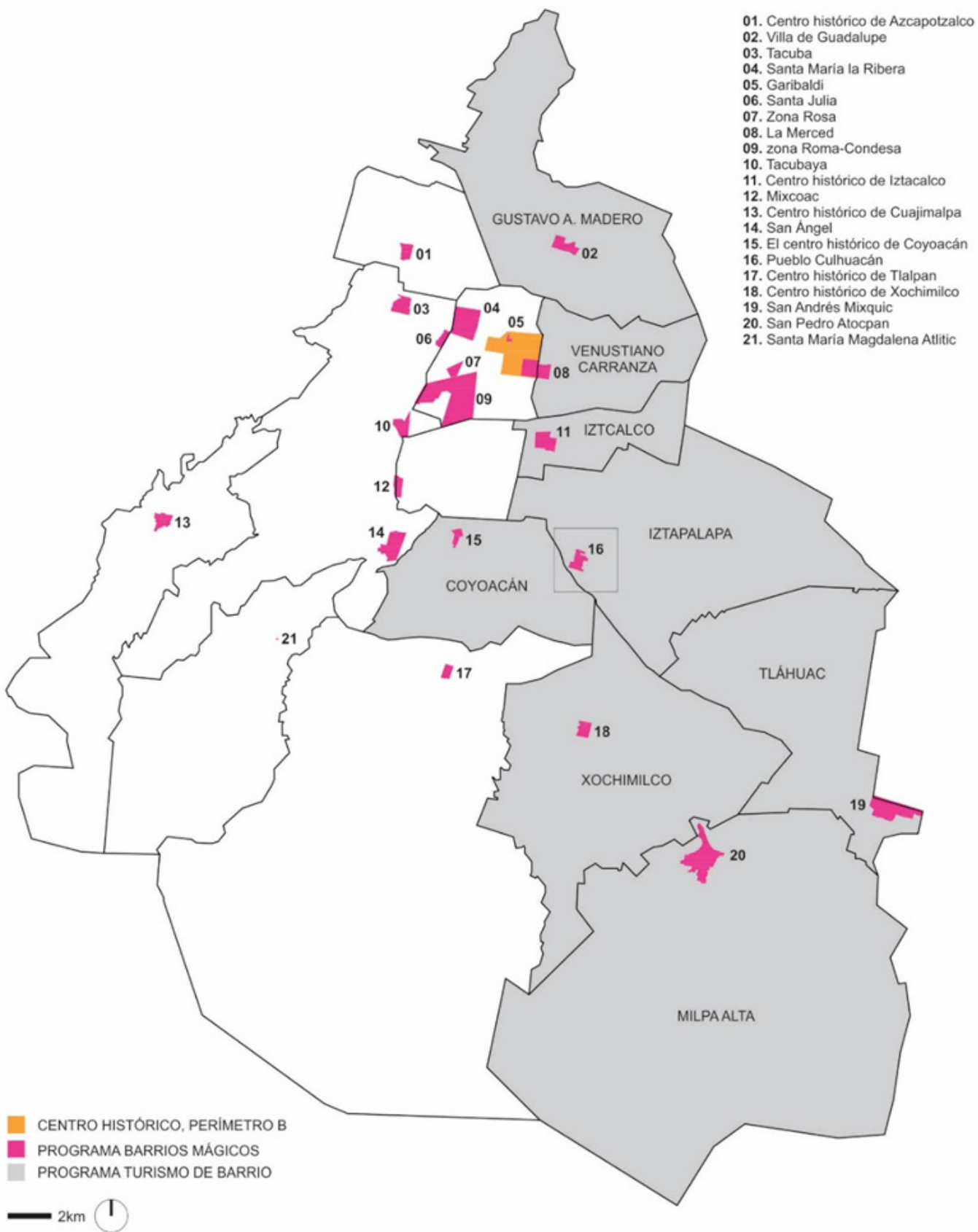

Fuente: elaboración propia.

de $77 \%$ nacionales contra $23 \%$ de extranjeros). El programa delega en la iniciativa particular de las alcaldías el desarrollo de obras de infraestructura y embellecimiento urbano de calles, banquetas y luminarias (Gómez Flores, 2019). ${ }^{12}$ 
En cuanto a estrategias puntuales, para el caso de Iztapalapa, por ejemplo, se habla de convertir la Central de Abasto (principal mercado de la zona metropolitana del Valle de México) en un espacio de congresos y convenciones, conjuntamente con la promoción de recorridos al Cerro La Estrella (escenario del evento de La Pasión de Cristo realizado cada año), del Festival de Tapetes Florales en Santa Cruz Meyehualco y del Carnaval de los Ocho Pueblos de Iztapalapa (Gómez Flores, 2019). Para Iztacalco, por su parte, se prevé la transformación de La Nueva Viga (mercado de pescados y mariscos, considerado el más grande de América Latina) en un corredor gastronómico a "completarse" con otros segmentos turísticos, contemplando además continuar con el rescate de la Ermita histórica, el área de conservación de Sierra Catarina y parques "habitados por indigentes" (Gómez Flores, 2019).

En este sentido, la estrategia territorial propuesta desde el DDPT apunta a sitios muy específicos, a través de polígonos de actuación que contengan hitos con fuerza barrial (ya sea la historia, festividades, atractivos materiales, etc.) y que permitan plantear recorridos en distancias caminables. Como ejemplo, se explicaba sobre el polígono en el sector de Culhuacán (Figura 2):

Estos eran pueblitos que se "comió" la ciudad y que tienen una gran tradición de fiestas o particularidades barriales. Culhuacán es bastante grande, pero los pueblos originarios son estos [señala sectores más pequeños]. Pero más allá de visitar un sitio, se trata de ofrecer experiencias. Mucho tiene que ver con la gastronomía o las festividades, fiestas patronales, y bueno, sí que haya un sitio físicamente ancla, que en este caso sería el ex convento de Culhuacán (Arq. Victor Salas Castilla, comunicación personal, 20 de junio de 2019).

\section{Figura 2: Ejemplo de polígono de actuación, Programa} Turismo de Barrio: Culhuacán, Ciudad de México.

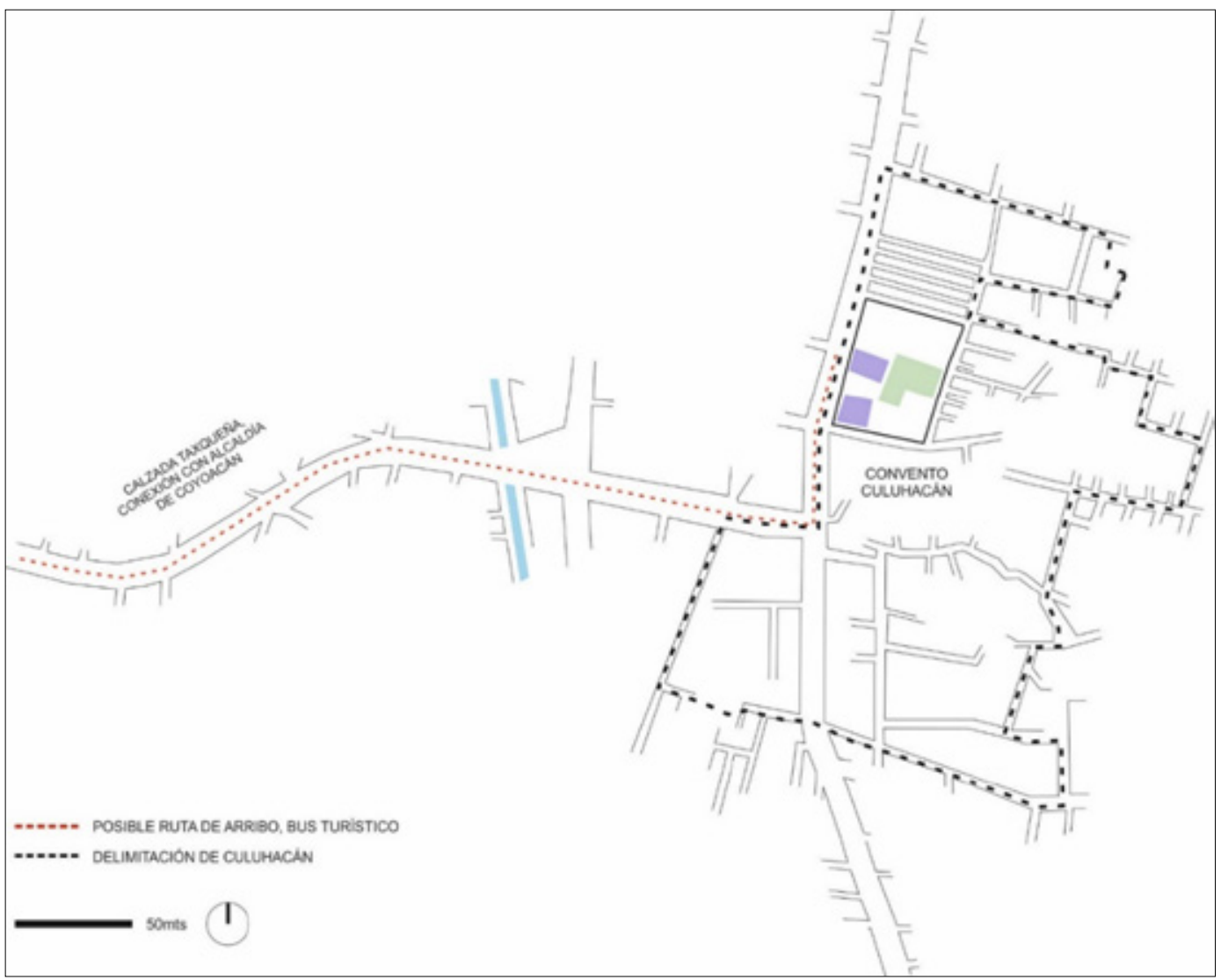

Fuente: elaboración propia en base al polígono exhibido en la entrevista al titular de la DDPT, SECTUR CDMX. 
A su vez, se plantea que los polígonos estén conectados por avenidas, permitiendo la movilidad entre unos y otros a través de la gestión de los tour-operadores, piezas clave para la puesta en marcha de las rutas, en la medida en que se trata de sectores que, en general, no cuentan con líneas de transporte público estructurales como metro o metro-bus. Cuando se consultó al titular de la DDPT sobre el trabajo con estos actores, explicó que:

Los tour-operadores fuertes aquí son Turibús, Capital Bus, y algunos otros más chicos. A ellos se les ocurre una ruta, por ejemplo, la de las Cantinas Sur, se platica y aquí se autoriza, porque tienen que tener el permiso de SECTUR, de la Secretaria de Movilidad y hasta de Obras Públicas (ellos les ayudan con la poda, los cables de las calles, etc.). Es una logística muy especial, que además se tiene que ir manteniendo (Arq. Victor Salas Castilla, Comunicación personal, 20 de junio de 2019).

El fomento de estas rutas se plantea, primeramente, de manera publicitaria, pero cuando comiencen a funcionar y puedan evaluarse las de mayor éxito, se prevé destinar fondos para su puesta en valor y embellecimiento urbano. Además, se desarrollarían guías, folletos y videos (que estén en redes sociales) para la promoción de estas rutas turísticas. De hecho, de acuerdo a la Lic. Riojas de la Peña, titular de la Dirección General del Instituto de Promoción Turística del SECTUR CMDX, el turismo de barrio ya forma parte de los diez segmentos turísticos a los que se orienta la promoción de la ciudad (naturaleza, médico, deportivo, cultural, gastronómico, LGTBIQ, lujo, reuniones, romance y barrio), por ello se buscará poner en valor las tradiciones y valores, artesanías y gastronomía de los sitios afectados, a partir de un inventario de recursos que se está desarrollando (Comunicación personal, 20 de junio de 2019).

\section{2. "Mi Barrio" y "Turismo a más Barrios" en Ciudad de Buenos Aires}

En el marco de actividades de divulgación de conocimiento, la red World Tourism Cities Federation $(\text { WTCF })^{13}$ en colaboración con la Organización Mundial del Turismo (UNWTO), presentó un manual sobre buenas prácticas de rendimiento turístico: City Tourism Performance Research (UNWTO/WTCF, 2017), en relación a ciertas ciudades de la red, incluida la Ciudad de Buenos Aires. Este manual constó de un monitoreo sobre el performance del turismo urbano (a través de visitas de campo, entrevistas a informantes claves y datos obtenidos de fuentes oficiales), con el objetivo de asistir a los creadores de políticas públicas de turismo en el desarrollo de acciones estratégicas para la gobernanza y manejo de la actividad turística, estableciendo recomendaciones generales que pudieran aplicarse en aquellos casos que pretendan ser competitivos en materia de turismo urbano (UNWTO/WTCF, 2017).

En ese contexto el ENTUR expuso el proyecto Mi Barrio (Figura 3). Presentado como un trabajo de investigación aplicada, constó del relevamiento de información en 20 barrios de la ciudad (al menos uno de cada Comuna) entre abril y septiembre de 2016, principalmente, a través de focus groups con los vecinos, entrevistas a informantes clave, observación etnográfica y fotografías. La finalidad que perseguía era "capturar la identidad" de cada uno de los barrios, identificando oportunidades para el desarrollo de rutas turísticas "originales y deferenciales" según el barrio, y así direccionar el flujo turístico hacia los mismos (Carlucci y Orlando, 2017).

Bajo la idea rectora de que "cada barrio tiene una esencia para mostrarle al mundo" y que los visitantes buscan "experiencias auténticas" cuando visitan las ciudades, el proyecto se presentó como la oportunidad para la creación de nuevos productos turísticos que diversificaran la oferta de la ciudad, a través de la incorporación de lugares fuera de los circuitos tradicionales (Carlucci y Orlando, 2017). En este sentido, los documentos presentados sobre el trabajo explicaban que "cuanto los vecinos más se apropien de los lugares destacados de sus barrios y los intervengan, más convocarán a los turistas del interior del país o del extranjero" (Carlucci y Orlando, 2017).

Si bien muchos de los barrios analizados pertenecían a sectores turísticos consolidados de la ciudad: microcentro, Balvarena, La Boca, San Telmo, Recoleta, Palermo y sus zonas aledañas (que ya vienen sufriendo el efecto del derrame comercial de sectores como Palermo Hollywood y Palermo Soho); barrios más alejados, localizados en la zona suroeste y sureste de la ciudad, eran presentados como factibles de convertirse en sitios turísticos.

Un ejemplo de ello, fueron las acciones específicas delineadas para Mataderos. Este tradicional barrio porteño, que recibió su nombre por los mataderos de ganado vacuno que abundaban en la zona hacia 1900, fue presentado como un lugar familiar, tranquilo y de casas bajas, pero con pérdida del protagonismo de su Mercado de Hacienda (tradicional centro comercializador de carne que abastece a la industria frigorífica de Buenos Aires) y decrecimiento de la actividad comercial, afectado también por 
Figura 3: Barrios afectados por el programa Mi Barrio.

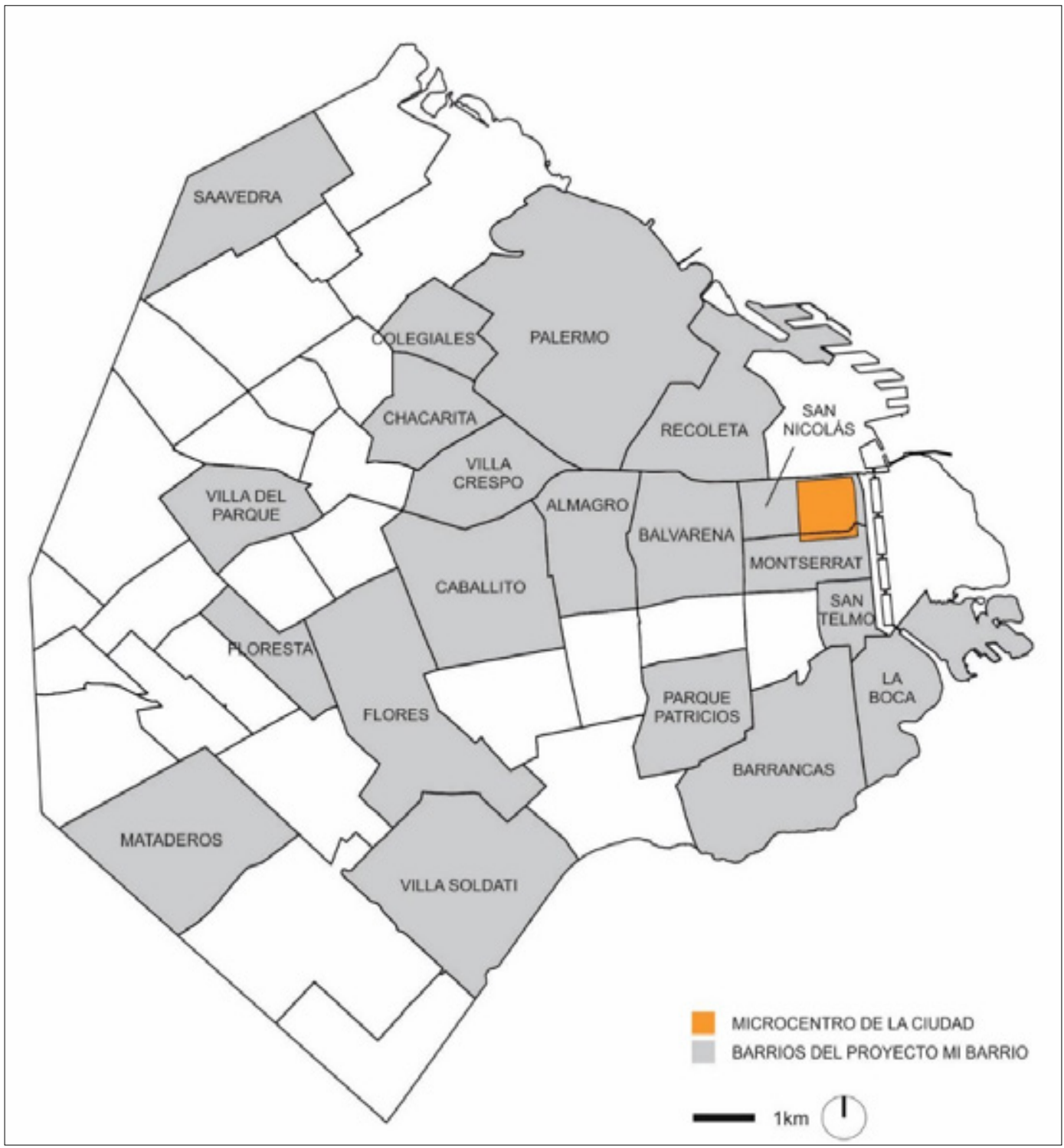

Fuente: elaboración propia en base a la información de documentos del programa Mi Barrio.

la inseguridad y las drogas. Ante este panorama, Mi Barrio proponía la oportunidad de devolverle la vitalidad perdida, capitalizando el proceso de promoción de la Feria de Mataderos (atracción turística ofertada por el ENTUR como "el costado más autóctono de la ciudad") ${ }^{14}$ e, incluso, a través del controvertido traslado del Mercado de Hacienda y posterior reconversión del predio. ${ }^{15}$ Con este proyecto el ENTUR proponía, a su vez, capitalizar el valor simbólico del tradicional predio para configurar un "eje cívico/turístico", que tendría como principales actividades la gastronomía y las compras, y permitiría la generación de empleos en rubros afines al turismo.

A pesar de la ambición de Turismo de Barrio, sólo se materializaron dos estrategias específicas relacionadas al proyecto. Por un lado, la apertura de un centro de atención turística en el tradicional barrio de San Nicolás. Por otro, la extensión de la ruta del Bus Turístico (puesto en marcha en 2009 y 
concesionado a una empresa privada) que, hacia principios de 2017, incorporó al sector de Palermo Soho en su recorrido. Concebido como un espacio que brinda posibilidades de consumo en una atmósfera con características de autenticidad fuera de los circuitos tradicionales (Rodríguez, 2018), la ruta del bus se reconfiguró con la incorporación de dos recorridos y dos paradas respectivamente: el outlet Distrito Arcos y Plaza Inmigrantes de Armenia, nuevo centro gastronómico del sector (Figura 4).

Como resultado del reporte elaborado por UNWTO/WTCF en relación a Mi Barrio, se afirmaba:

El proyecto ha ayudado a identificar áreas de intervención que necesitan mejoras sociales, urbanas, económicas y turísticas, fomentando así la inclusión social, el espíritu empresarial comercial y turístico y el desarrollo sostenible. Asimismo, ha permitido identificar las áreas sobreexplotadas que pueden descongestionarse, redistribuyendo beneficios y mejorando el funcionamiento del destino en su conjunto. (UNWTO/WTCF, 2017:48).

Conforme a entrevistas realizadas a funcionarios del ENTUR durante octubre de 2019, Turismo de Barrio no continuó desarrollándose. Sin embargo, un análisis de la oferta turística actual, a través de la página de turismo de la Ciudad, presenta un novedoso apartado: Turismo por Barrios, ${ }^{16}$ indicando una serie de atractivos a visitarse en todos los barrios de la Ciudad bajo el título "Explorá cada Barrio" y "Espacios culturales, obras de arte urbano y la variada oferta gastronómica son sólo el principio de los innumerables atractivos que ofrecen los 48 barrios porteños. Elegí un barrio, descubrí como un local la identidad de cada uno y iconocé lo más auténtico de Buenos Aires!" (ENTUR, s.f.).

Figura 4: Cambio en el recorrido del Bus Turístico, Ciudad Autónoma de Buenos Aires.

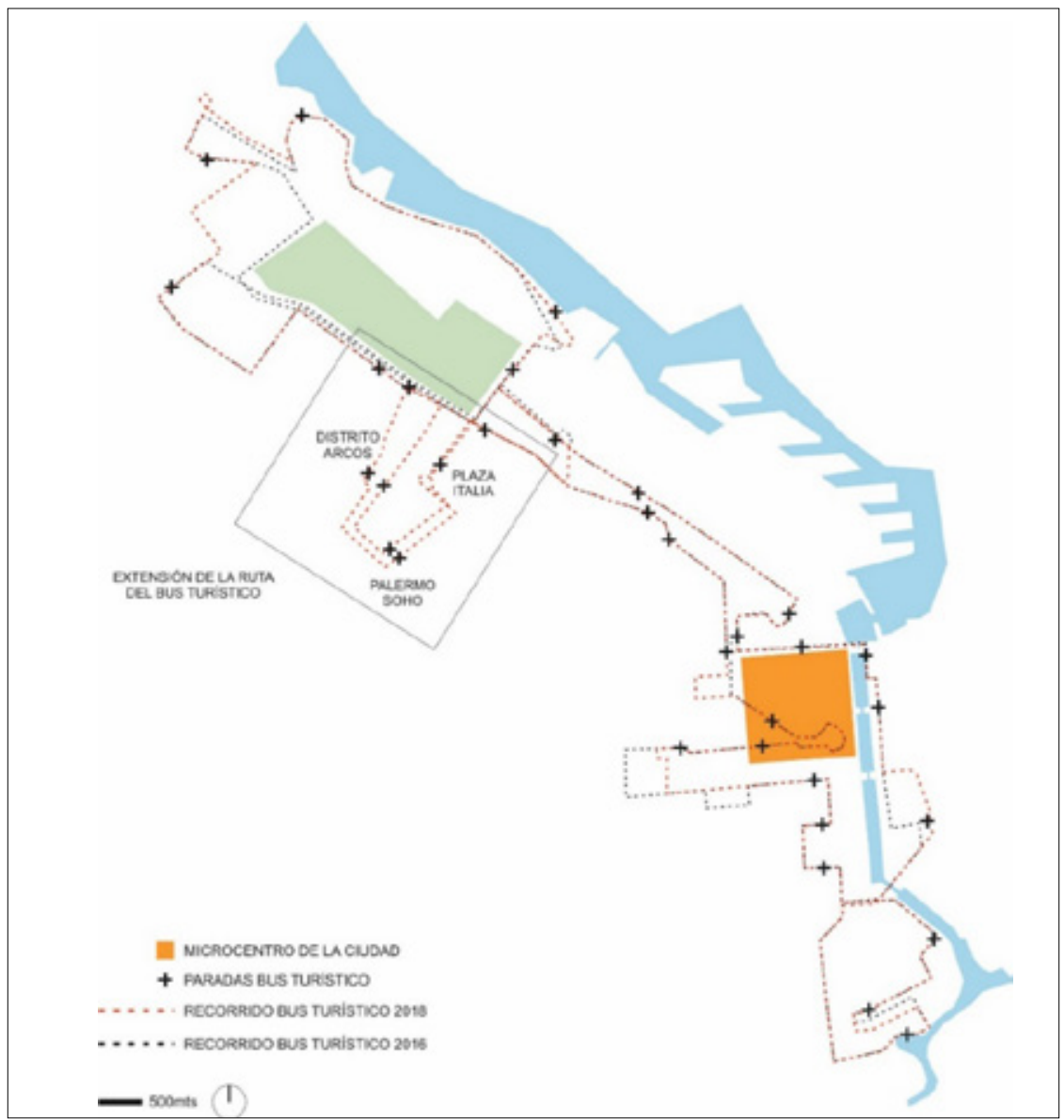

Fuente: elaboración propia. 
De acuerdo a la Lic. Lucila Wernli, Gerente Operativa de Herramientas Digitales y Desarrollo de la Oferta, dependiente de la Dirección General de Desarrollo Turístico del ENTUR, este mapa, que se armó para empezar a promocionar a los barrios geolocalizando sus atractivos turísticos, es parte de un plan más ambicioso, pensado a cuatro años, llamado Turismo a más Barrios (Comunicación personal, 04 de octubre de 2019). Respecto a su continuidad con el proyecto de Mi Barrio, la funcionaria comentaba que se trata de una actualización, ya que la ciudad se transformó mucho desde 2016, y cuestiones que en los focus groups se apuntaban (por ejemplo, el problema de la inseguridad), ya fueron resueltas. Entonces, era necesario salir nuevamente a identificar las condiciones de los barrios y la percepción actual de los vecinos.

Finalmente, cuando se consultó por qué era deseable llevar el turismo a los barrios de la Ciudad, ella comentó:

Hoy nosotros tenemos un corredor muy marcado [en referencia al sector norte de la Ciudad], un corredor turístico en donde solamente se generan beneficios para sus habitantes. Entonces, lo que nosotros buscamos es, y como en líneas generales las tendencias del turismo se están orientando a esto, distribuir los ingresos económicos en la medida que sea posible (...). A alguien se le ocurrió "a más barrios", que no es turismo "a todos los barrios", sino que es diversificar la oferta. Es encontrar aquellos barrios que tienen potencialidad, que están dispuestos a recibir turistas y trabajar con ellos para que hoy el ingreso económico que, por ejemplo, llega a Palermo, llegue también a Chacarita, a Villa Crespo, a Colegiales. Que se expanda un poco más. Entonces generas beneficios para una red mucho más amplia (...). Todo eso con el objetivo de que lo que hoy deja el turismo en la ciudad no quede solamente en siete barrios del corredor, sino que se derrame y genere un beneficio para el comerciante que invirtió en ese bar en Caballito [hace referencia a un ejemplo que apuntó con anterioridad, un barrio poco promocionado a nivel turístico] (Lic. Lucila Wernli, Comunicación personal, 04 de octubre de 2019).

\section{Resultados}

De acuerdo a la información expuesta, podemos trazar semejanzas y un posible hilo conductor entre los instrumentos y las estrategias adoptadas en ambos casos de estudio (Figura 5). Por un lado, el marco legal general en el que se inscriben las acciones -leyes nacionales- continúa legitimando el discurso positivo asociado al turismo, relacionando su fomento con tres ejes de mejoras: económicas, sociales y culturales. Apoyados en estas leyes, los planes nacionales y programas sectoriales (específicos para la actividad en el caso de Ciudad de México y estratégicos generales para Buenos Aires) presentan a la diversificación de la oferta como un objetivo específico clave para un desarrollo exitoso y para el logro del fortalecimiento de la competitividad turística (meta general presente, además, en las leyes locales de turismo). En ambos casos, esa diversificación no sólo se traduce en una oferta temática diversa, sino también en una distribución territorial equitativa o equilibrada en el ámbito de las ciudades, asociada a la descongestión de ciertas áreas o la idea de expandir o replicar sus beneficios hacia otros sectores.

En este sentido, las leyes de turismo locales desarrollan un instrumento específico, las zonas o lugares de desarrollo prioritario, asociadas a rasgos naturales, históricos o culturales singulares, que puedan capitalizarse para su futura conversión en atractivo turístico.

Por otro lado, a nivel de los programas locales, los objetivos mencionados encuentran su correlato territorial en la unidad de los barrios. ${ }^{17}$ En congruencia con lo postulado en las leyes nacionales, estos programas presentan al turismo como una actividad capaz de generar beneficios como la creación de empleos, desarrollo de un tejido empresarial, derrame económico y mejora de la calidad de vida de la población local y del espacio urbano. Todo ello, a través del desarrollo de experiencias auténticas que capitalizan una identidad barrial asociada a la forma de vida de sus habitantes y a su sentido de pertenencia con el lugar. A su vez, la información recabada cristalizó cuatro estrategias de acción para estos programas (con diferentes acentos según el caso de estudio), involucrando a vecinos, sector público y privado, con distinto tipo de participación:

1) Oferta ligada a actividades barriales. El vecino del barrio es el creador de la oferta a partir de su propio trabajo, lo que supone su desplazamiento desde la esfera cotidiana hacia el ámbito del turismo, proceso en el que el Gobierno actúa como capacitador o asesor técnico. Para el caso de Ciudad de Buenos Aires, además, la metodología de recolección de información es explícitamente bottom-up.

2) Embellecimiento urbano e infraestructura. Tareas de mejora del espacio público (infraestructura de servicios, luminaria, espacios de permanencia, etc.) que son desarrolladas por las Alcaldías en Ciudad de México o del Gobierno de la Ciudad en Buenos Aires.

3) Acceso y movilidad. La llegada de turistas es gestionada por empresas privadas de transporte, a través del diseño de rutas turísticas específicas. En este caso el Gobierno genera las condiciones para la circulación y la promoción. 
4) Estrategias puntuales de envergadura que apuntalan el proceso. Activación de grandes equipamientos urbanos, por ejemplo, la refuncionalización de Mercados existentes, que sirven como atractores para el establecimiento de polos comerciales en el área circundante. Aunque no es explícito, esto supondría la asociación público-privada (sino la acción del sector privado de manera exclusiva) liderando el proceso.

Figura 5: Elementos comunes a los instrumentos y estrategias analizadas para las Ciudades de México y Buenos Aires.

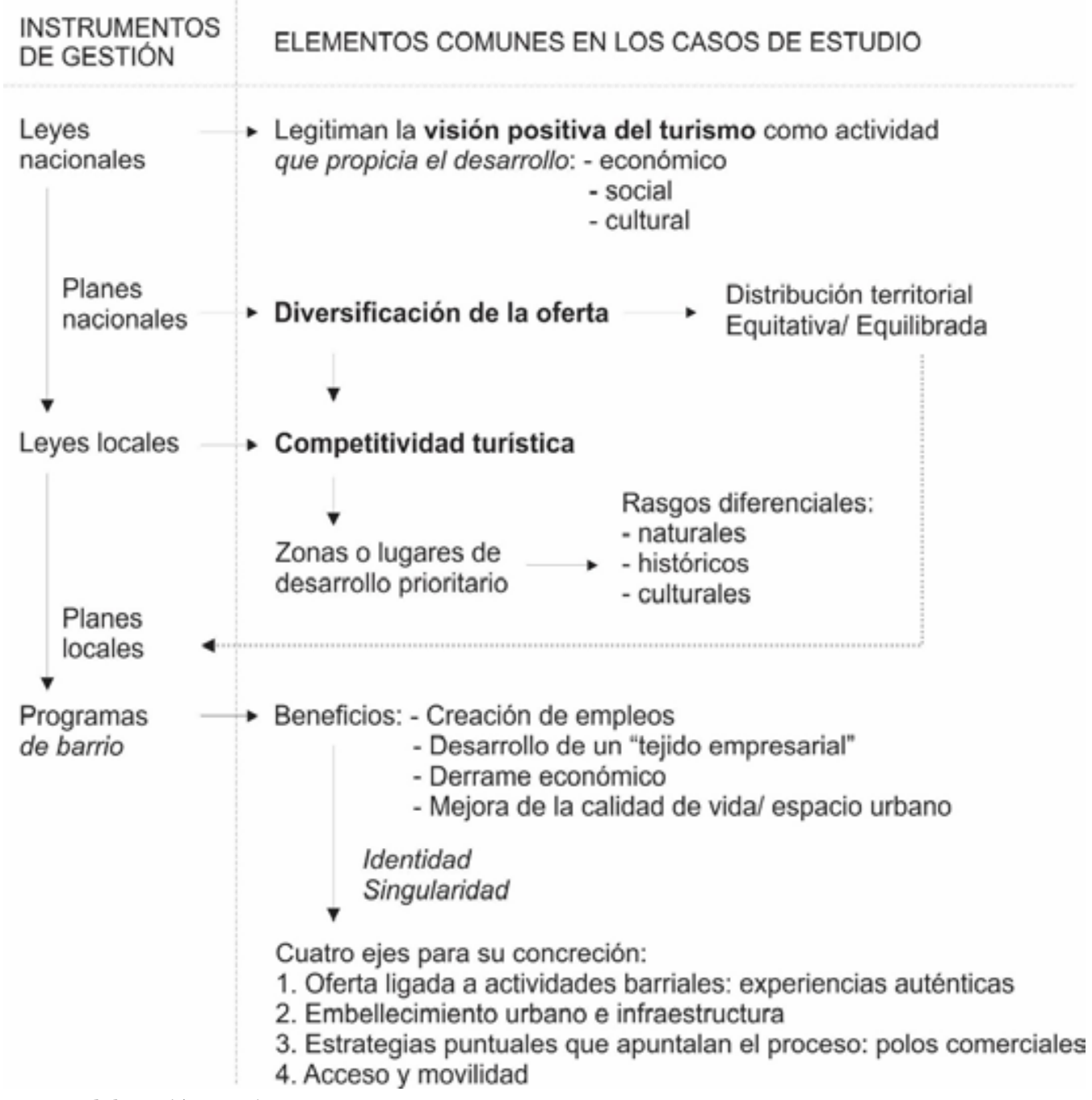

Fuente: elaboración propia.

\section{Conclusiones}

El artículo discutió cómo, en el marco de modelos de gobernanza de criterios empresariales, la actividad del turismo es concebida como un medio de competitividad entre países o ciudades y como un recurso que posibilita el desarrollo económico, social y cultural, entre otras acciones, a partir de la diversificación de la oferta turística. Orientándose al público que busca experiencias auténticas en los 
lugares que visita, la descentralización de la oferta, fuera de los sitios o circuitos turísticos tradicionales, se presenta como un medio para conseguirlo. Un fenómeno abordado por estudios académicos desde el lado de la demanda (especialmente a partir de la motivación de turistas, residentes y trabajadores), pero escasamente desde el lado de la oferta (específicamente, desde el rol que las políticas públicas pueden tener en su desarrollo) en el ámbito de las ciudades latinoamericanas.

Bajo esta línea, y en el marco de la flexibilización de los instrumentos de la política pública -que permiten la adaptación a los escenarios cambiantes del mundo globalizado-, encontramos que las Ciudades de México y Buenos Aires están adoptando estrategias discursivas y materiales similares en relación al desarrollo del turismo. Esta convergencia, pese a sus diferentes orientaciones políticas, confirma la existencia de un modelo de gestión que se impone sobre las particularidades locales, haciendo prevalecer lineamientos comunes.

Con esto nos referimos al desarrollo de un discurso coherente acerca de los aspectos positivos del turismo, que actúa como un gran paraguas con capacidad de albergar una serie de propuestas diversas dada su generalidad. Bajo el mismo, en las estrategias de barrio desarrolladas en las Ciudades de México y Buenos Aires, el Gobierno parece adquirir el rol de promotor de oferta privada-individuos y empresas-, en la medida en que se captan e incentivan actividades surgidas de ese ámbito. Apoyándose en discursos que apelan a la competitividad turística a través de una distribución territorial equitativa de la oferta (premisas de la planificación estratégica), el barrio se vuelve el escenario de autenticidad y singularidad necesario para el desarrollo de experiencias que conecten a la ciudad con los gustos de los visitantes, a través de la participación e involucramiento de los vecinos (supuestos beneficiarios del proceso). Paralelamente, sin embargo, se contemplan acciones de otra escala e impacto, como el desarrollo de rutas turísticas o polos comerciales, que tendrían como desarrolladores a empresas privadas, acompañadas por acciones de reacondicionamiento urbano por parte del sector público.

Esto conduce a pensar que la premisa de diversificación de la oferta podría estar actuando como un mecanismo más de valorización urbana, teniendo al Estado como posibilitador (con reducción de su intervención directa) y al sector privado como desarrollador, en el marco de una clara desregularización de la actividad turística: ausencia de instrumentos normativos que generen obligaciones al cumplimiento de los programas, falta de documentos oficiales que indiquen, por ejemplo, como se adjudican los nombramientos y los recursos de los mismos, o evaluaciones de programas predecesores, etc. De esta manera, el trabajo manifiesta como las nuevas estrategias de turismo urbano podrían estar contribuyendo al desarrollo de políticas neoliberales orientadas a la valorización de sitios alejados de los circuitos tradicionales y el importante rol que el Estado tiene en ese proceso.

\section{Bibliografía}

Almirón, A.; Bertoncello, R.; Kuper, D. y Ramírez, L. 2008. El "turismo como impulsor del desarrollo" en Argentina. Una revisión de los estudios sobre la temática. Aportes y Transferencias, 12(1), 57-86.

Balslev Clausen, H. y Velázquez, M. 2013. El turismo cultural en Argentina, México y Brasil. Avances y desafíos. Diálogos Latinoamericanos, 21, 9-30.

Barbini, B., Biasone, A., Cacciutto, M., Castellucci, D., Corbo, Y. y Roldán, N. 2011. Gobernanza y Turismo: Análisis del Estado del Arte. Simposio Internacional Gobernanza y Cambios Territoriales: experiencias comparadas de migración de amenidad en las Américas. Pucón, Chile.

Baum, T. 1994. The development and the implementation of national tourism policies. Tourism Management, 15(3), 185-192.

Borja, J. y Castells, M. 1997. Local y global. Madrid: Taurus

Bowman, K. 2014. Policy Choice, Social Structure, and International Tourism in Buenos Aires, Havana, and Rio de Janeiro. Latin American Research Review, 50(3), 135-156.

Bowman, K. 2013. Peddling Paradise: The Politics of Tourism in Latin America. Boulder: Lynne Rienner Publishers.

Britton, S. 1991. Tourism, capital, and place: towards a critical geography of tourism. Environment and Planning, D Society and Space, 9, 461- 478.

Cócola-Gant, A. 2015. Tourism and commercial gentrification. RC21 International Conference on "The Ideal City: between myth and reality. Representations, policies, contradictions and challenges for tomorrow's urban life".

Cohen, E. 1988. Authenticity and commodification in tourism. Annals of Tourism Research, 15, 371-386. 
Delgadillo, V. 2014. Urbanismo a la carta: teorías, políticas, programas y otras recetas urbanas para ciudades latinoamericanas. Cadernos Metrópole, 16(31), 89-111.

Delgadillo, V., Díaz, I. y Salinas, L. (Coord.) 2015. Perspectivas del estudio de la gentrificación en México y América Latina. México: UNAM, Instituto de Geografía.

Dinardi, C. 2015. Citied for sale: Contesting city branding and cultural policies in Buenos Aires. Urban Studies, 54(1), 85-101.

Fernández Güell, J. M. 1997. Planificación estratégica de ciudades. Barcelona: Gustavo Gilli.

Gómez, M. y Zunino Singh, D. 2008. La (re) valorización de la zona sur y su patrimonio histórico cultural como recurso turístico. En: Hilda Herzer (Comp.) Barrios al Sur. Renovación y pobreza en la ciudad de Buenos Aires (pp. 325-367). Argentina: Café de las Ciudades.

Harvey, D. 1998. La condición de la posmodernidad. Investigación sobre los orígenes del cambio cultural. Buenos Aires: Amorrourtu. (Edición original: 1990).

Harvey, D. 1989. From Managerialism to Entrepreneurialism: The Transformation in Urban Governance in Late Capitalism. Geografiska Annaler, 71(1), 3-17.

Hall, M. 2000. Tourism Planning. Policies, processes and relationships. Essexs: Prentice Hall.

Hiernaux, D. y González, C. 2014. Turismo y gentrificación: pistas sobre una articulación. Revista de Geografía Norte Grande, 58, 55-70.

Jacint, J. 2011. El análisis de las policy networks: ¿Una nueva perspectiva sobre la relación entre políticas públicas y Estado? En Jefatura de Gabinete de Ministros (Comp.), Lecturas sobre el Estado y las Políticas Públicas: Retomando el debate de ayer para fortalecer el actual (pp. 505-526). Buenos Aires: Proyecto de Modernización del Estado Jefatura de Gabinete de Ministros de la Nación.

Janoschka, M., Sequera J., y Salinas, L. 2014. Gentrificación en España y América Latina: Un diálogo crítico. Revista de geografía Norte Grande, 58, 7-40.

Jessop, B. 2002. Liberalism, Neoliberalism, and Urban Governance: A State-Theoretical Perspective. En: Neil Brenner y Nik Theodore (Coords.), Spaces of Neoliberalism: Urban Restructuring in North America and Western Europe (pp. 104-125). Nueva Jersey: Blackwell.

Kanai, M. y Ortega-Alcázar, I. 2009. The Prospects for Progressive Culture- Led Urban Regeneration in Latin America: Cases from Mexico City and Buenos Aires. International Journal of Urban and Regional Research, 33(2), 483-501.

Ley de Turismo de la Ciudad Autónoma de Buenos Aires, $N^{\circ}$ 600/0. Legislatura de la Ciudad Autónoma de Buenos Aires, 07 de junio de 2001.

Ley de Turismo del Distrito Federal. Gaceta Oficial del Distrito Federal, Distrito Federal, México, 30 de agosto de 2010.

Ley Federal de Turismo. Presidencia de la República, México, 32 de diciembre de 1992.

Ley Nacional de Turismo, №25997. Senado y Cámara de Diputados de la Nación Argentina, Boletín Oficial, 07 de enero de 2005.

Maitland, R. y Newman, P. 2009. World tourism cities: developing tourism off the beaten track. Londres: Routledge.

Maitland, R. 2008. Conviviality and Everyday Life: the Appeal of New Areas of London for Visitors. International Journal of Tourism Reasearch, 10, 15-25.

Ministerio de Economía y Finanzas GCBA. (s.f.). Pernoctaciones de turistas no residentes por destino. Aeropuerto Internacional de Ezeiza y Aeroparque Jorge Newbery. Años 2005/4to. trimestre 2018. Recuperado de: https://www.estadisticaciudad.gob.ar/eyc/?cat=142

Novy, J. 2011. Marketing Marginalized Neighborhoods. Tourism and Leisure in the $21^{\text {st }}$ Century Inner City. Tesis de Doctorado. Universidad de Columbia.

Reglamento de la Ley de Turismo del Distrito Federal. Ciudad de México, 29 de marzo de 2012.

Rodriguez, L. 2019. Experiencias de consumo como nuevos productos turísticos. La promoción de festivales y eventos en la Ciudad Autónoma de Buenos Aires. Revista de Urbanismo, 40, 1-16.

Rodriguez, L. 2018. Palermo Viejo: Palermo Soho. Los nuevos espacios de consumo como insumo del turismo urbano. En: J. Mansilla y C. Milano (Coord.), Ciudad de Vacaciones. Conflictos urbanos en espacios turísticos, (pp. 289-326). Barcelona: OACU-Pol.len.

Rodríguez, M., Arqueros Mejica, S., Rodríguez, F., Gómez Schettini, M. y Zapata, M. 2011. La política urbana 'pro': continuidades y cambios en contextos de renovación en la Ciudad de Buenos Aires. Cuaderno Urbano: espacio, cultura y sociedad, 11(11), 101-121.

Sánchez Salazar, M. T. y Gutiérrez de MacGregor, M. T. (Coords,). 2016. Espacio y Ocio: El turismo en México. Colección Temas Selectos de Geografía de México. México DF: Atlántida Coll-Hurtado. 
Schenkel, E. 2017. Política turística y turismo social. Una perspectiva latinoamericana. Buenos Aires: Ediciones CICCUS.

Secretaría de Turismo. 2005. Plan Federal Estratégico de Turismo Sustentable.

SECTUR. 2013_a. Programa Sectorial de Turismo 2013-2018. Diario oficial.

SECTUR. 2013_b. Programa Sectorial de Turismo 2013-2018. Ciudad de México.

SECTUR DF. 2011. $5^{\circ}$ Informe de Gobierno, Secretaría de Turismo del Distrito Federal. Ciudad de México.

Smith, N. 1996. The new urban frontier. Gentrification and the revanchist city. Londres y nueva York: Routledge.

Vainer, C. 2000. Pátria, empresa e mercadoria. Notas sobre a estratégia discursiva do planejamento estratégico. En: Arantes, O., Vainer, C. y Maricato, E. A cidade do pensamento único. Desmanchando consensos (pp. 75-104). Petrópolis: Ed. Vozes.

Velasco, M. 2011. La política turística. Una arena de acción autónoma. Cuadernos de Turismo, 27, 953-969.

Wallingre, N. (Comp.) 2017. Desarrollo del turismo en América Latina: fases, enfoques e internacionalización. Bernal: Universidad Nacional de Quilmes.

\section{Recursos electrónicos}

Carlucci, L. y Orlando, M. 2017. Mi Barrio y El Matadero. Taller Técnico: "Estudio OMT - WTCF sobre Rendimiento del Turismo Urbano". Recuperado de: http://marketintelligence.unwto.org/es/event/ taller-tecnico-estudio-omt-wtcf-sobre-rendimiento-del-turismo-urbano

Gómez Flores, C. 10 de enero de 2019. Con "Turismo de Barrio" promueven visitas a Iztacalco e Iztapalapa. La Jornada. Recuperado de: https://www.jornada.com.mx/ultimas/2019/01/10/con-2018turismo-de-barrio2019-promueven-visitas-a-iztacalco-e-iztapalapa-848.html?fbclid=IwAR2EhCtsqSnYhqkLI0 jq-T-H3iHZ7bGEIIBvq0yKgovBBaVeQ-gDGRDgwcU

ENTUR. s.f.. ¡Explorá cada barrio! Recuperado de <https://turismo.buenosaires.gob.ar/es/turismo-por-barrios $>$

INPROTUR. s.f.. Marca Pais. Recuperado de <http://www.turismo.gov.ar/inprotur>

Rammsy, D. 08 de abril de 2019. Turismo de Barrio y Día de Muertos, los atractivos para potenciar en la CDMX. Publimetro. Recuperado de: https://www.publimetro.com. mx/mx/destacado-tv/2019/04/07/ turismo-barrio-dia-muertos-los-atractivos-potenciar-la-cdmx.html

UNWTO/WTCF. 2017. UNWTO/WTCF City Tourism. Performance Research. Report for Case Study: "Buenos Aires, Argentina". Recuperado de: http://marketintelligence.unwto.org/es/event/taller-tecnico-estudio-omt-wtcf-sobre-rendimiento-del-turismo-urbano

WTCF. 2019. About WTCF. Recuperado de: http://en.wtcf.org.cn/About/WhoWeAre/

\section{Notas}

1 Cabe recordar que, aunque el desarrollo de la actividad turística se presente como positiva en términos discursivos dentro de la esfera pública (justificada en la teoría económica del efecto de derrame), también comporta una serie efectos cuestionables. Dinámicas interrelacionadas de desplazamientos simbólicos (Janoschka et al., 2014), comerciales (Cócola-Gant, 2015) y de población residente (Hiernaux y Gonzalez, 2014; Delgadillo et al., 2015), son algunos de los efectos más evidentes en destinos turísticos consolidados, pero también en aquellos en vías de consolidación.

2 Dinardi (2015) desarrolla esta idea para el caso de la "marca ciudad" de Buenos Aires, mientras que Rodriguez (2019) lo hace específicamente en relación con el desarrollo de Festivales y Eventos de la misma ciudad.

3 En tanto construcción social y no como una cualidad medible de lo que se visita, resultado de creencias, ideologías, contexto, etc. (Cohen, 1988).

4 En 1997 los ciudadanos del Distrito Federal pudieron elegir a sus autoridades ejecutivas. Posteriormente, en enero de 2016, se produce la reforma política del Distrito Federal, modificación legislativa con la que la ciudad obtuvo un estatus político semejante al de las entidades federativas del país, cambiando su nombre a Ciudad de México. Por su parte, después de la reforma constitucional de 1994 que permitió la autonomía de la ciudad de Buenos Aires (hasta entonces regida por el gobierno nacional), en el año 1996 sus ciudadanos pudieron elegir Jefe de Gobierno.

5 Documentos del poder Ejecutivo, donde se establecen los objetivos y estrategias para el desarrollo del país, comprendiendo el período de cada mandato presidencial.

6 La denominación "CDMX" surge en 2016, por parte de las autoridades locales, como una estrategia de marketing orientada al desarrollo de la nueva marca-ciudad.

7 En 2011 se hizo una primera actualización del plan, extendiendo su plazo a 2020, mientras que en 2014 se aprobó la segunda actualización extendiendo el plazo a 2025.

8 En el momento de su creación, el ENTUR pertenecía al ámbito de la Secretaría General y de Relaciones Internacionales, luego pasó al Ministerio de Cultura, posteriormente al Ministerio de Modernización, Innovación y Tecnología, retornando en la actualidad a la primera Secretaría, después de la reducción de la cartera Ministerial de 2018. 
9 Son previos a estos planes el Plan Estratégico de Cultura de Buenos Aires 2001, el Plan Estratégico de Marketing Turístico 2001-2003 y el Plan de Marketing Turístico "Más Buenos Aires” publicado en 2007.

10 "Diseñado para promover el turismo cultural, independientemente de la especialización turística de cada Estado, buscando impulsar la imagen urbana, y las ciudades que tengan un patrimonio histórico y arquitectónico" (Velázquez García, 2013: 102).

11 La entrevista a funcionarios del área de turismo de Ciudad de México indicó la discontinuidad del programa a partir del último cambio de gestión. No se hallaron diagnósticos o evaluaciones sobre sus resultados.

12 La financiación del proyecto provendría, mayormente, del impuesto por hospedaje administrado por el Fondo Mixto de Promoción Turística -Mackinlay mencionó que 15 millones de pesos mexicanos irían destinados al programa-, la Secretaría de Desarrollo Económico (SEDECO) y recursos del Fondo para el Desarrollo Social (FONDESO) entre otras.

13 Guiada por la visión "el turismo hace la vida en la ciudad mejor", esta asociación no gubernamental y sin fines de lucro, tiene por objetivo el desarrollo de la industria del turismo a través de la cooperación y consultoría técnica entre sus miembros: ciudades turísticas y empresas (WCTF, 2019).

14 Ver promoción del Ente de Turismo de la Ciudad: https://turismo.buenosaires.gob.ar/es/atractivo/feria-de-mataderos

15 En 2017 el Gobierno de la Ciudad firmó un acuerdo para el desalojo del Mercado de Mataderos, amparado en el vencimiento de la prórroga de concesión del uso de las tierras y en la idea de poner en valor la zona. Aunque, primeramente, la sede se iba a ubicar en un terreno fiscal del partido de La Matanza, finalmente, se instalará en un predio privado del partido de Cañuelas. Recién en noviembre de 2019 se sancionó el proyecto del traslado, para la conversión del predio en un parque temático relacionado a la actividad rural, reubicándose allí la Feria de Mataderos. El gobierno podrá otorgar concesiones de uso, explotación y de obra pública, las que deberán ser cubiertas en un mínimo de $20 \%$ por los vecinos de la comuna. Esto implicará, además, la relocalización de las familias que allí habitan.

16 Ver: https://turismo.buenosaires.gob.ar/es/turismo-por-barrios

17 La distribución territorial de estos barrios merece un trabajo mas exhaustivo que permita superponer a los mapas desarrollados otras políticas urbanas, a fin de determinar las lógicas que subyacen en su selección. 